\title{
Two-Dimensional Decaying Elastoinertial Turbulence
}

\author{
J. J. J. Gillissen $\odot^{*}$ \\ Department of Mathematics, University College London, Gower Street, London, WC1E 6BT, United Kingdom
}

(Received 4 March 2019; revised manuscript received 20 August 2019; published 4 October 2019)

\begin{abstract}
We numerically simulate two-dimensional, decaying elastoinertial turbulence using the finitely extensible, nonlinear, elastic spring model. We vary the polymer concentration over seven decades, and observe two turbulent elastoinertial regimes. In the weakly coupled regime only the small scale structures change, while in the strongly coupled regime all structures change. This regime is dominated by elastoinertial shock waves with drag reduction properties; i.e., the energy decay rate decreases when the polymer concentration increases.
\end{abstract}

DOI: 10.1103/PhysRevLett.123.144502

There is great scientific interest in the fluid mechanics of polymer solutions, and in particular in the context of turbulent drag reduction in pipe flow [e.g., [1,2]]. This so-called elastoinertial turbulence, which is governed by a combination of inertial and elastic stresses, is not to be confused with elastic turbulence, which is governed by a combination of viscous and elastic stresses [e.g., [3] ]. One may distinguish two regimes of elastoinertial turbulence [4]. At modest polymer concentration, the turbulence changes only at the large wave numbers (small scales), while, at a large polymer concentration, the turbulence changes over the entire wave number range. This strongly coupled elastoinertial turbulence has universal features and, in pipe flow, this state is known as Virk's maximum drag reduction asymptote [5]. The characteristics of elastoinertial turbulence depend on the dimensionality. In Newtonian (polymer free) 3D turbulence there is a downscale energy cascade, i.e., a transport of inertial energy from large to small scales. In the weakly coupled regime, the polymers weaken this downscale cascade, enhancing the energy at the large scales, and reducing the energy at the small scales [6-8]. In the strongly coupled regime, the downscale energy cascade regains strength, reducing the energy at the large scales and enhancing the energy at the small scales [9]. In 2D, on the other hand, the energy cascade in the Newtonian regime is upscale, i.e., from small to large scales [10]. In the weakly coupled regime, the polymers suppress the upscale energy cascade, with a reduction of the energy at the large scales and an increase at the small scales [11-13] To our knowledge, strongly coupled elastoinertial turbulence has not yet been studied in 2D.

To shed light on this regime, we numerically simulate elastoinertial decaying 2D turbulence, using the finitely extensible nonlinear elastic (FENE) spring model [14]. We vary the polymer concentration over seven decades, and study the turbulence properties in both the weakly and the strongly coupled regimes. We thereby show that, in both regimes, the energy cascade is downscale over the entire wave number range. We furthermore show that the strongly coupled regime is dominated by elastoinertial shock waves, with drag reduction characteristics; i.e., the energy decay rate decreases when the polymer concentration increases. It is emphasized that decaying elastoinertial turbulence sustains over a long timescale (compared to the eddy turn over time), which is markedly different from elastic turbulence, which decays rapidly, and requires an external forcing to sustain $[15,16]$.

The FENE spring model reads [14]:

$$
\begin{aligned}
\boldsymbol{\nabla} \cdot \boldsymbol{u} & =0 \\
\partial_{t} \boldsymbol{u}+\boldsymbol{u} \cdot \boldsymbol{\nabla u}+\nabla p-\nu \nabla^{2} \boldsymbol{u} & =\nu \alpha \lambda^{-1} \nabla \cdot[s \boldsymbol{c}], \\
\partial_{t} \boldsymbol{c}+\boldsymbol{u} \cdot \boldsymbol{\nabla} \boldsymbol{c}-\boldsymbol{\nabla} \boldsymbol{u}^{T} \cdot \boldsymbol{c}-\boldsymbol{c} \cdot \boldsymbol{\nabla} \boldsymbol{u}= & \lambda^{-1}[\boldsymbol{\delta}-s \boldsymbol{c}] \\
& -\kappa \Delta x^{4} \sqrt{\boldsymbol{E}: \boldsymbol{E}} \nabla^{4} \boldsymbol{c} .
\end{aligned}
$$

Here $\nu=\eta / \rho$ is the solvent kinematic viscosity, $\eta$ is the solvent dynamic viscosity, $\rho$ is the fluid mass density, $p$ is the fluid pressure divided by $\rho, \boldsymbol{E}=\frac{1}{2}\left(\boldsymbol{\nabla} \boldsymbol{u}+\boldsymbol{\nabla} \boldsymbol{u}^{T}\right)$ is the rate of strain tensor, $\boldsymbol{u}$ is the fluid velocity vector, $\boldsymbol{c}$ is the nondimensional polymer conformation tensor [14], $s=$ $[1-\operatorname{tr}(\boldsymbol{c}) / b]^{-1}$ is the nonlinear spring constant, $\alpha \sim n R_{G}^{3}$ is the polymer coil volume fraction at equilibrium, $R_{G}$ is the coil size at equilibrium, $n$ is the polymer number density, $\lambda \sim \rho \nu R_{G}^{3} /\left(k_{B} T\right)$ is the polymer relaxation time, $k_{B} T$ is the Boltzmann energy, $b \sim\left(l / R_{G}\right)^{2}$ is the extensibility parameter, $l$ is the polymer contour length, $\kappa$ is a nondimensional prefactor, $\kappa \Delta x^{4} \sqrt{\boldsymbol{E}: \boldsymbol{E}}$ is the artificial hyper diffusivity, $\Delta x=L_{x} / N_{x}$ is the grid spacing, $L_{x}$ is the domain size, and $N_{x}$ is the number of grid points per dimension. By fixing the magnitude of $\kappa \sim 1$, the artificial hyper diffusion term $-\kappa \Delta x^{4} \sqrt{\boldsymbol{E}: \boldsymbol{E}} \nabla^{4} \boldsymbol{c}$ suppresses the $\boldsymbol{c}$ modes with wavelengths that are comparable or smaller than $\Delta x$; i.e., it ensures that the numerical scheme produces a well-resolved solution. 
Equation (1) is simulated on a biperiodic domain. The numerical method has been used in Refs. [17,18], and is described in Sec. S4 in the Supplemental Material [19], which includes Ref. [20]. The domain size is $L_{x}=2 \pi$ and, unless states otherwise, we use $N_{x}^{2}=128^{2}$ grid points and a viscosity of $\nu=1 \times 10^{-3}$. The artificial hyper diffusivity is $\kappa=0.2$. The relaxation time is $\lambda=20$, which in the Newtonian flow at $t=4$ corresponds to a Weissenberg number of $\mathrm{We}=\lambda \sqrt{\langle\boldsymbol{E}: \boldsymbol{E}\rangle} \approx 50$, where $\langle\cdot\rangle$ is the spatial average. The extensibility parameter is $b=10$, which corresponds to semiflexible polymer chains, and the concentration parameter is varied between $\alpha=10^{-1}$ and $10^{6}$. We introduce the fluid elasticity parameter $\mathcal{E}=\nu \alpha /\left(\lambda \mathcal{U}^{2}\right)$, which is the order of magnitude estimate of the ratio of the elastic energy $P=-\frac{1}{2} \nu \alpha b \lambda^{-1} \log [1-\operatorname{tr}(\boldsymbol{c}) / b] \sim \nu \alpha \lambda^{-1}$ and the inertial energy $K=\frac{1}{2} \boldsymbol{u}^{2} \sim \mathcal{U}^{2}$, where $\mathcal{U}$ is the rms of the velocity at $t=0$. Based on these parameters, $\mathcal{E}$ is varied between $5 \times 10^{-6} \leq \mathcal{E} \leq 5 \times 10^{1}$. Depending on the value for $\mathcal{E}$ the flow is either in the Newtonian $(N)$, weakly coupled $(W)$, strongly coupled $(S)$, or laminar $(L)$ regime. Although we demarcate these different flow regimes with vertical dashed lines in Figs. 3 and 4 below, there are no sharp boundaries between $N, W, S$, and $L$, and these dashed lines are to be interpreted as smooth transitions.

The initial conditions at $t=0$ are an equilibrium polymer conformation $\boldsymbol{c}=\boldsymbol{\delta}$ and a random velocity field $\boldsymbol{u}$, where the energy is concentrated in the modes with $|\boldsymbol{k}| \leq 8$. The initial velocity field is normalized, such that $\mathcal{U}=\sqrt{\left\langle\boldsymbol{u}^{2}\right\rangle}=1$ at $t=0$. After starting the simulation, the initial, random configuration develops into a turbulent state. During the initial transient, the polymers adsorb a portion of the initial $K$. We artificially force the system with a body force $\sim(\boldsymbol{u} / \Delta t)\left[1 /\left\langle\boldsymbol{u}^{2}\right\rangle-1\right]$ between $0<t<t_{F}$, where $t_{F}=1$ is the forcing time, such that $\left\langle\boldsymbol{u}^{2}\right\rangle=1$ during $0<t<t_{F}$, while the polymers absorb energy. Here $\Delta t$ is the computational time step. At $t=t_{F}$ the forcing is switched off, and the system is let to decay. Figure 1 shows the resulting time development of several wave number components of the spectra of $K$ and of $P$ for $S$ with $\mathcal{E}=4 \times 10^{-1}$. At $t=0, K$ is concentrated at $|\boldsymbol{k}| \leq 8$. As
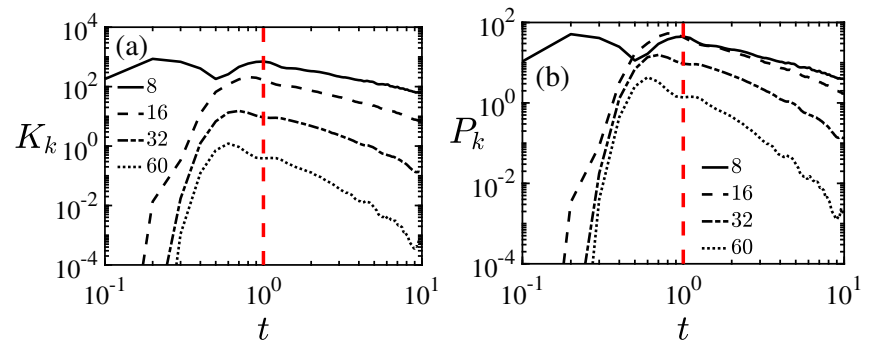

FIG. 1. The time development of several wave number components (legend) of the spectra of the inertial energy (a) and of the elastic energy (b) for $S$ with $\mathcal{E}=4 \times 10^{-1}$. The red dashed lines mark the end of the forcing and the start of the free decay. time progresses, $K$ is transformed into $P$, and for short times $t \leq 0.3, P$ is concentrated at $|\boldsymbol{k}| \leq 8$. At larger times, turbulence sets in, which transfers energy from large to small scales. The spectra develop over a characteristic timescale, which is of the order of $t_{F}=1$ (red dashed lines), at which instant the forcing is switched off and the system is let to decay.

In addition to the $128^{2}$ simulations we have conducted simulations using $1024^{2}$ grid points, a solvent viscosity of $\nu=5 \times 10^{-5}$, and a forcing period of $t_{F}=2$, for $N$ with $\mathcal{E}=0, W$ with $\mathcal{E}=5 \times 10^{-4}, S$ with $\mathcal{E}=5 \times 10^{-1}$, and $L$ with $\mathcal{E}=5 \times 10^{1}$, while the other parameters $\kappa=0.2$, $b=10$, and $\lambda=20$ are unchanged. The vorticity fields at $t=4$ for these high resolution simulations are plotted in Fig. 2. In the $N$ simulation, we observe the usual large-scale vortex structures. The vorticity in $W$ is similar, although somewhat modified, with the emergence of filamentlike structures, also observed in [11]. In $S$ there is a complete alteration of the turbulence, with the emergence of elastoinertial shock waves; see Movie S1 in [19]. These shocks travel normal to the fluid velocity vector, and share similarity but differ in details with the longitudinal elastoinertial shocks, which were predicted in Ref. [21]. In $L$ the vortical structures do not evolve and remain identical to those at $t=0$.

The equations for $K$ and for $P$ read [20]:

$$
\partial_{t} K+\boldsymbol{u} \cdot \boldsymbol{\nabla}(K+p)=\boldsymbol{\nabla} \cdot(\boldsymbol{\sigma} \cdot \boldsymbol{u})-D-T,
$$
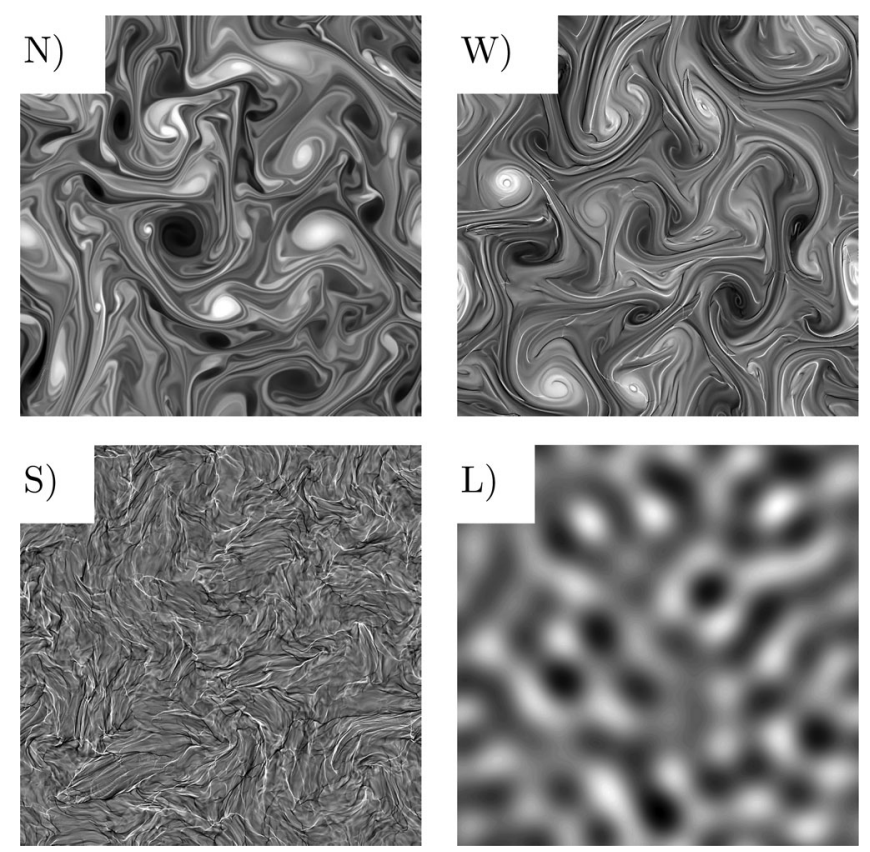

FIG. 2. Vorticity field at $t=4$ in high resolution simulations ( $1024^{2}$ grid points), corresponding to Newtonian flow $(N)$ with $\mathcal{E}=0$, weakly coupled flow $(W)$ with $\mathcal{E}=5 \times 10^{-4}$, strongly coupled flow $(S)$ with $\mathcal{E}=5 \times 10^{-1}$, and laminar flow $(L)$ with $\mathcal{E}=5 \times 10^{1}$. 
and:

$$
\partial_{t} P+\boldsymbol{u} \cdot \boldsymbol{\nabla} P=T-R,
$$

where $\sigma=\nu \alpha \lambda^{-1} s \boldsymbol{c}$ is the polymer stress tensor divided by $\rho, D=\nu \nabla \boldsymbol{u}: \nabla \boldsymbol{u}$ is the viscous dissipation of $K, T=$ $\nabla u: \sigma$ is the transformation between $K$ and $P$, and $R=$ $\frac{1}{2} \lambda^{-1} \operatorname{tr}(\boldsymbol{\sigma})[1-\operatorname{tr}(\boldsymbol{c}) / b]^{-1}$ is the dissipation of $P$ through polymer relaxation.

Figure 3(a) shows the spatial mean (solid lines) and the spatial standard deviation (dotted lines) of $K$ and $P$ as functions of $\mathcal{E}$ at $t=4$, and Fig. 3(b) shows the corresponding data for $T, D$, and $R$. In $N\left(\mathcal{E} \lesssim 5 \times 10^{-4}\right), K$ does not change upon increasing $\mathcal{E}$ and $P \ll K$, in $W$ $\left(5 \times 10^{-4} \lesssim \mathcal{E} \lesssim 10^{-2}\right) K$ decreases with $\mathcal{E}$ and $P<K$, in $S$ $\left(10^{-2} \lesssim \mathcal{E} \lesssim 5 \times 10^{-1}\right) K$ increases with $\mathcal{E}$ and $P>K$, and in $L\left(5 \times 10^{-1} \lesssim \mathcal{E}\right) K$ does not change upon increasing $\mathcal{E}$ and $P \gg K$. In both $W$ and $S$ the decay is dominated by $R$, while $D \ll R$; i.e., the polymers absorb energy from the flow, and dissipate this via chain relaxation. In $S$ the spatial standard deviation of $T$ is several orders of magnitude larger than its spatial mean.

To study the physics of $T$, we develop an analytical model for the elastoinertial shear waves (Sec. S1 in [19]). The model shows that on average $P \approx 3 K$, and that $K$ and $P$ are roughly in phase with each other while they lag $T$ by
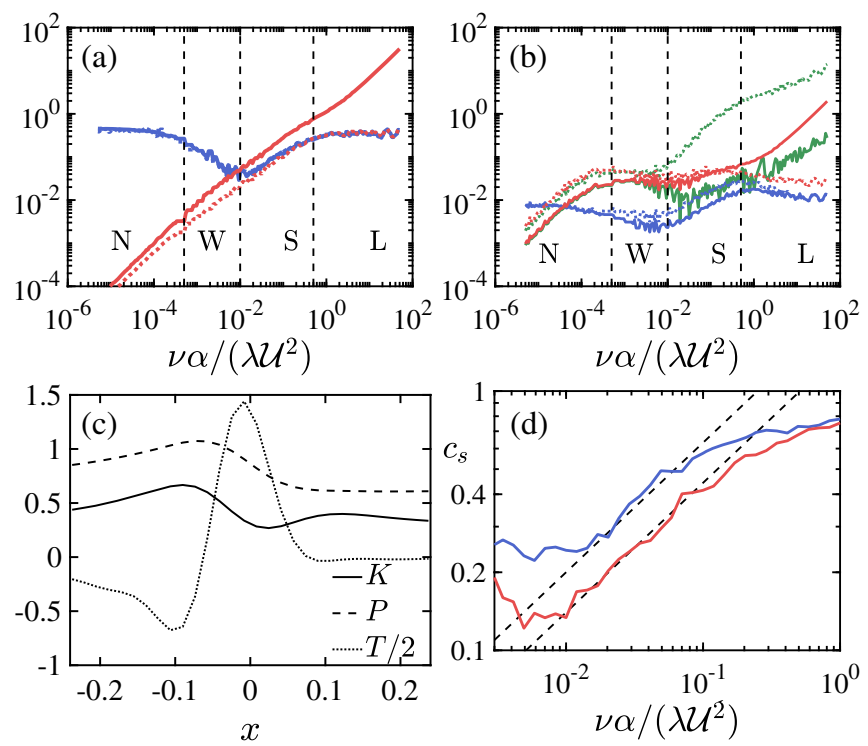

FIG. 3. (a) Spatial mean (solid lines) and spatial standard deviation (dotted lines) of inertial energy $K$ (blue) and elastic energy $P$ (red) at $t=4$. (b) Spatial mean (solid lines) and spatial standard deviation (dotted lines) of viscous dissipation $D$ (blue), polymer relaxation $R$ (red) and transfer $T$ from $K$ to $P$ (green) at $t=4$. (c) Profiles of $K, P$, and $T$ across the elastoinertial shock waves for $S$ with $\mathcal{E}=4 \times 10^{-1}$ at $t=4$. The depicted conditionally averaged wave travels from left to right. (d) Wave speed $c_{s}$ at $t=4$ (blue) and $t=8$ (red). The dashed lines correspond to the theoretical prediction $c_{s} \sim(\nu \alpha / \lambda)^{1 / 2}$. $\pi / 2$. To verify the significance of this model, we develop an algorithm to extract, from the simulation data, the profiles of fluid mechanical variables across the elastoinertial shock waves (Sec. S3 in [19]). Figure 3(c) shows the resulting profiles of $K, P$, and $T$ for $S$ with $\mathcal{E}=4 \times 10^{-1}$ at $t=4$. $D$ and $R$ are orders of magnitude smaller than $T$ and are not shown. The conditionally averaged wave, depicted in Fig. 3(c), travels from left to right. The $T$-wave consists of a high and narrow crest followed by a shallow and wide trough. We furthermore see that $P \approx 2 K$ and that $K$ and $P$ are roughly in phase with each other while they lag $T$ by $\pi / 2$ in qualitative agreement with the analytical model. The agreement confirms that the observed flow structures in $S$ (Movie S1 in [19]) are elastoinertial shock waves.

To further study the elastoinertial nature of the shock waves observed in Movie S1 in [19], we extract the wave speed $c_{s}$ from the simulation data using $c_{s}=\left[\left\langle\partial_{t}^{2} \Omega \partial_{x}^{2} \Omega\right\rangle /\right.$ $\left.\left\langle\left(\partial_{x}^{2} \Omega\right)^{2}\right\rangle\right]^{1 / 2}$, which is derived in Sec. S2 in [19], and where $\Omega=\partial_{x} u_{y}-\partial_{x} u_{x}$ is the fluid vorticity. Figure 3(d) shows the resulting $c_{s}$ as a function of $\mathcal{E}$ at $t=4$ (blue) and $t=8$ (red). For $10^{-2} \lesssim \mathcal{E} \lesssim 10^{-1}$ the wave speed agrees with the analytical model $c_{s} \sim(\nu \alpha / \lambda)^{1 / 2}$ (dashed lines), which is derived for linear springs (Sec. S1 in [19]). For $\mathcal{E} \gtrsim 10^{-1}$, the spring stiffness reduces together with the polymer extension [Fig. 4(c)], which explains the deviation of the simulation data from the theoretical prediction.
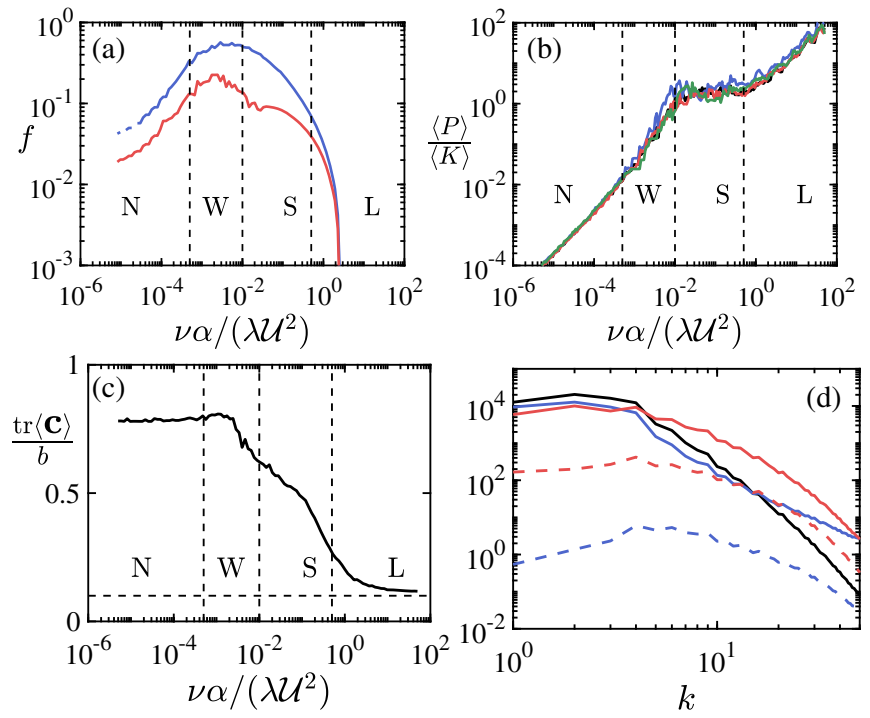

FIG. 4. (a) Energy decay rate between $t=1$ and $t=5.5$ (blue) and between $t=5.5$ and $t=10$ (red). (b) Ratio of elastic energy and inertial energy at $t=4$ using a $N_{x}^{2}=128^{2}$ grid and a forcing period of $t_{F}=1$ (black), $\left(t, N_{x}, t_{F}\right)=(4,256,1) \quad($ red $)$, $\left(t, N_{x}, t_{F}\right)=(8,128,1) \quad$ (blue), and $\left(t, N_{x}, t_{F}\right)=(5,128,2)$ (green). (c) Polymer extension at $t=4$. The horizontal dashed line corresponds to the equilibrium configuration. (d) Spectral density at $t=4$ of inertial energy (solid lines) and elastic energy (dashed lines) using a $128^{2}$ grid. The black, blue, and red curves correspond to $N$ with $\mathcal{E}=0, W$ with $\mathcal{E}=6 \times 10^{-4}$, and $S$ with $\mathcal{E}=4 \times 10^{-1}$, respectively. 
Assuming an exponential decay of the total (inertial plus elastic) energy $\langle K\rangle+\langle P\rangle \sim \exp (-f t)$, we compute the decay rate as $f=\log \left[\left(\langle P\rangle_{i}+\langle K\rangle_{i}\right) /\left(\langle P\rangle_{f}+\langle K\rangle_{f}\right)\right] /\left(t_{f}-t_{i}\right)$, where $\langle K\rangle_{i}$ and $\langle P\rangle_{i}$ are $\langle K\rangle$ and $\langle P\rangle$ at $t=t_{i}$, and $\langle K\rangle_{f}$ and $\langle P\rangle_{f}$ are the corresponding values at $t=t_{f}$. Figure 4(a) shows the resulting $f$ as a function of $\mathcal{E}$ for two intervals $\left(t_{i}, t_{f}\right)=(1,5.5)$ and $\left(t_{i}, t_{f}\right)=(5.5,10)$, respectively. The decay is faster in the earlier interval, but the qualitative behavior of $f(\mathcal{E})$ is identical for both intervals.

The maximum decay rate of $f \approx 0.5$ occurs in $W$ at $\mathcal{E} \approx 3 \times 10^{-3}$, which corresponds to a minimum characteristic decay time of $f^{-1} \approx 2$. In Figs. 3 and 4 we consider flow statistics at $t=4$, which is sufficiently large that the flow has developed structures over the full wave number spectrum (see Fig. 1), and which is sufficiently small that the energy at $t=4$ is at least $14 \%$ of the energy at $t=t_{F}$.

In $S$ the decay rate decreases while increasing the polymer concentration, which is equivalent to a drag reduction effect. Similar as in maximum drag reduction in turbulent boundary layers $[5,22]$, this drag reduced state has universal features, where the corresponding flow structures are independent of the polymer concentration.

This is shown in Fig. 4(b) by the (nearly) constant $\langle P\rangle /\langle K\rangle$ as a function of $\mathcal{E}$ in $S$. In that figure we compare data at $t=4$ using a $N_{x}^{2}=128^{2}$ grid and a forcing period of $t_{F}=1$ (black), $\left(t, N_{x}, t_{F}\right)=(4,256,1)($ red $),\left(t, N_{x}, t_{F}\right)=$ $(8,128,1)$ (blue), and $\left(t, N_{x}, t_{F}\right)=(5,128,2)$ (green). The close agreement between these four data sets demonstrates that the $128^{2}$ grid is sufficient to capture the correct physics, and that the findings of this Letter do not sensitively depend on the choices for $t$ and $t_{F}$.

We plot in Fig. 4(d) the wave number $k$ spectrum of $K$ and of $P$ at $t=4$, using a grid of $128^{2}$. These results agree well with those on a $256^{2}$ grid, which are shown in Sec. S5 in [19]. The agreement confirms that the $128^{2}$ grid is sufficient to capture the correct physics. For $W$ with $\mathcal{E}=$ $6 \times 10^{-4}$ (blue lines), the $K$ spectrum is enhanced only for relatively large wave numbers $(k>20)$, while it is hardly affected for smaller $k$. For $S$ with $\mathcal{E}=4 \times 10^{-1}$ (red lines) on the other hand, the $K$ spectrum is enhanced over nearly the entire $k$ range $(k>4)$. Interestingly, in $S$ the shape of the $K$ spectrum approaches that of $P$ over the entire $k$ range.

In Sec. S6 in [19], which includes Ref. [23], we compute the interscale transfer of $K$ due to inertial stress $I_{\ell}$ and due to polymer stress $T_{\ell}$. For $N T_{\ell}=0$ and $I_{\ell}$ corresponds to an upscale energy cascade. For $W$ the upscale effect of $I_{\ell}$ is weakened, and overwhelmed by the downscale effect of $T_{\ell}$. For $S$ the cascade is still downscale, but with a reduced strength, as compared to $W$.

In summary, we have numerically demonstrated that, at large polymer concentrations, 2D elastoinertial turbulence consists of elastoinertial shock waves, which transport energy from large to small scales. This regime has drag reduction characteristics; i.e., the energy decay rate decreases when the polymer concentration increases.

We would like to acknowledge financial support from the Engineering and Physical Sciences Research Council of the United Kingdom Grant No. EP/N024915/1.

*jurriaangillissen@gmail.com

[1] D. Samanta, Y. Dubief, M. Holzner, C. Schäfer, A. N. Morozov, C. Wagner, and B. Hof, Elasto-inertial turbulence, Proc. Natl. Acad. Sci. U.S.A. 110, 10557 (2013).

[2] G. H. Choueiri, J. M. Lopez, and B. Hof, Exceeding the Asymptotic Limit of Polymer Drag Reduction, Phys. Rev. Lett. 120, 124501 (2018).

[3] A. Groisman and V. Steinberg, Elastic turbulence in a polymer solution flow, Nature (London) 405, 53 (2000).

[4] E. Balkovsky, A. Fouxon, and V. Lebedev, Turbulence of polymer solutions, Phys. Rev. E 64, 056301 (2001).

[5] P. S. Virk, Drag reduction fundamentals, AIChE J. 21, 625 (1975).

[6] J. M. J. Den Toonder, M. A. Hulsen, G. D. C. Kuiken, and F. T. M. Nieuwstadt, Drag reduction by polymer additives in a turbulent pipe flow: Numerical and laboratory experiments, J. Fluid Mech. 337, 193 (1997).

[7] E. van Doorn, C. M. White, and K. R. Sreenivasan, The decay of grid turbulence in polymer and surfactant solutions, Phys. Fluids 11, 2387 (1999).

[8] E. De Angelis, C. M. Casciola, R. Benzi, and R. Piva, Homogeneous isotropic turbulence in dilute polymers, J. Fluid Mech. 531, 1 (2005).

[9] Y. Dubief, V. E. Terrapon, and J. Soria, On the mechanism of elasto-inertial turbulence, Phys. Fluids 25, 110817 (2013).

[10] R. H. Kraichnan, Inertial ranges in two-dimensional turbulence, Phys. Fluids 10, 1417 (1967).

[11] G. Boffetta, A. Celani, and S. Musacchio, Two-Dimensional Turbulence of Dilute Polymer Solutions, Phys. Rev. Lett. 91, 034501 (2003).

[12] H. Kellay, Polymers suppress the inverse transfers of energy and the enstrophy flux fluctuations in two-dimensional turbulence, Phys. Rev. E 70, 036310 (2004).

[13] Y. Jun, J. Zhang, and X.-L. Wu, Polymer Effects on Smalland Large-Scale Two-Dimensional Turbulence, Phys. Rev. Lett. 96, 024502 (2006).

[14] R. B. Bird, R. C. Armstrong, and O. Hassager, Dynamics of Polymeric Liquids (John Wiley and Sons, New York, NY, USA, 1987).

[15] S. Berti, A. Bistagnino, G. Boffetta, A. Celani, and S. Musacchio, Two-dimensional elastic turbulence, Phys. Rev. E 77, 055306(R) (2008).

[16] S. Berti and G. Boffetta, Elastic waves and transition to elastic turbulence in a two-dimensional viscoelastic Kolmogorov flow, Phys. Rev. E 82, 036314 (2010).

[17] J. J. J. Gillissen, A. Vilquin, H. Kellay, R. Bouffanais, and D. K. P. Yue, A space-time integral minimisation method for the reconstruction of velocity fields from measured scalar fields, J. Fluid Mech. 854, 348 (2018). 
[18] J. J. J. Gillissen, R. Bouffanais, and D. K. P. Yue, Data assimilation method to de-noise and de-filter particle image velocimetry data, J. Fluid Mech. 877, 196 (2019).

[19] See the Supplemental Material at http://link.aps.org/ supplemental/10.1103/PhysRevLett.123.144502 for a movie of the turbulent, elostoinertial shock waves, an analytical model for the shock waves, an algorithm to measure the speed of the shock waves, an algorithm to measure the variation of fluid mechanical variables across the shock waves, a numerical method to simulate FENE-turbulence, a comparison of energy spectra at different grid resolutions and an analysis of the inter-scale energy transfer.
[20] P. K. Ptasinski, B. J. Boersma, F. T. M. Nieuwstadt, M. A. Hulsen, B. H. A. A. Van den Brule, and J.C. R. Hunt, Turbulent channel flow near maximum drag reduction: Simulations, experiments and mechanisms, J. Fluid Mech. 490, 251 (2003).

[21] T. Odijk, Turbulent drag reduction in one and two dimensions, Physica (Amsterdam) 298A, 140 (2001).

[22] J. J. J. Gillissen, Maximum drag reduction simulation using rodlike polymers, Phys. Rev. E 86, 046304 (2012).

[23] M. K. Rivera, W. B. Daniel, S. Y. Chen, and R. E. Ecke, Energy and Enstrophy Transfer in Decaying TwoDimensional Turbulence, Phys. Rev. Lett. 90, 104502 (2003). 